\title{
ALIMENTAÇÃO DE OLIGOSARCUS LONGIROSTRIS MENEZES \& GĖRY (OSTEICHTHYES, ACESTRORHYNCHINAE) DO RESERVATÓRIO DE SALTO SEGREDO, PARANÁ, BRASIL
}

\author{
Ana Maria Gealh ${ }^{1}$ \\ Norma Segatti Hahn ${ }^{2}$
}

\begin{abstract}
Feeding of Oligosarcus longlrostris Menezes \& Gèry (OstelCHTHYES, ACESTrorhynchinae) OF the Salto SEgredo Reservoir, PARANÁ, Brazil. In the feeding study of $O$. longirostris Menezes \& Gèry, 1983, 211 stomachs from fish caught between March 1993 and February 1994 were analysed. Diet composition was analysed by the index of relative importance which deals with the numerical and gravimetric occurrence frequency. Results indicated that the species is piscivorous and its preferential food consisted of Astyanax sp. 2. It was also found that Astyanax sp. 3 became an important food item, depending on the site of capture and its availability. Availability of prey seems to have a strong influence on the diet of $O$. longirostris. This same tendency has been observed during the different seasons of the year. Ontogenetic changes have also been found in the basic diet.

KEY WORDS. Oligosarcus longirostris, diet, piscivorous, Salto Segredo Reservoir
\end{abstract}

A saicanga, Oligosarcus longirostris Menezes \& Gèry, 1983 é um peixe de distribuição restrita, sendo capturado apenas no rio Iguaçu acima das Cataratas. No reservatório de Salto Segredo foi a sexta espécie mais abundante na pesca experimental (AgostinHo et al. 1997).

A ecologia trófica de peixes tem sido objeto de múltiplos estudos, pois através dela pode-se inferir acerca da auto-ecologia de uma espécie em particular, bem como da estrutura trófica de determinada comunidade. Muitas espécies de peixes estão adaptadas a consumir um tipo específico de alimento, mas podem ocorrer variações na dieta relacionadas com o crescimento ou idade do peixe (NIKOLSKY 1963). Este trabalho pretende contribuir para o conhecimento do espectro alimentar desta espécie, bem como suas variações temporais, espaciais e ontogenéticas na dieta.

\section{MATERIAL E MÉTODOS}

\section{Descrição da área de estudos}

O reservatório de Salto Segredo localiza-se no rio Iguaçu, na divisa dos municípios de Pinhão e Mangueirinha, Paraná, cerca de $2 \mathrm{~km}$ a montante do rio Jordão, próximo ao Arroio da Divisa, nas coordenadas $25^{\circ} 47^{\prime}$ S e $52^{\circ} 07^{\prime} \mathrm{W}$. Concluído em outubro de 1992 sua área é de $83 \mathrm{~km}^{2}$ e a profundidade máxima de 108 $\mathrm{m}$, sendo o tempo de detenção hidráulica de 50 dias.

1) Depto de Biologia Geral, Universidade Estadual de Ponta Grossa. Praça Santos Andrade, 84010-790 Ponta Grossa, Paraná, Brasil.

2) Nupelia, Universidade Estaudal de Maringá. Avenida Colombo 5790, 87020-900 Maringá, Paraná, Brasil. 
Onze estações de coletas foram estabelecidas para estudos da ictiofauna local (Fig. 1), sendo três no corpo principal do reservatório [Barragem (Barr), Linígrafo (Lini), Areia (Arei)], três nos tributários da margem direita [rios Verde (Verd), São Pedro (Sped) e Floresta (Flor)] e três da margem esquerda [rios Covó (Covó), Butiá (Buti), e Iratim (Irar)], e uma estação a jusante da barragem (Jusa).

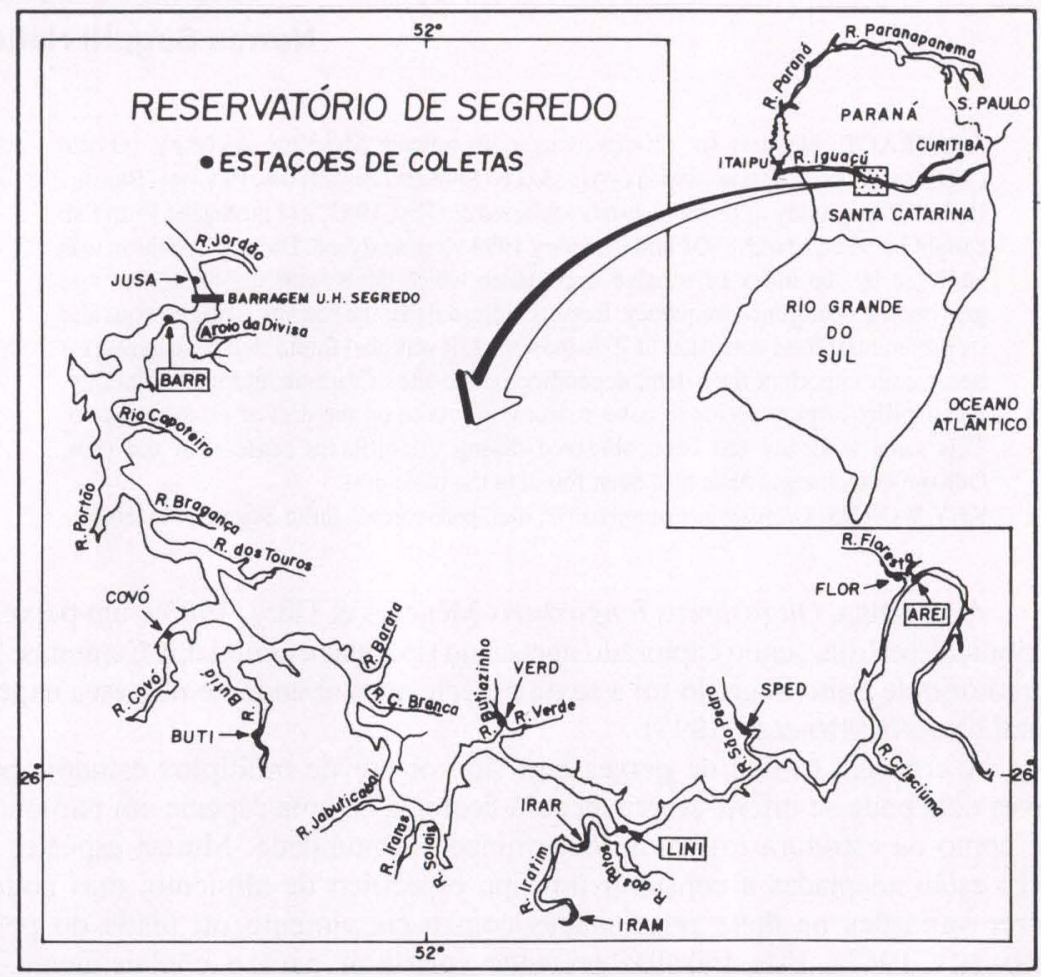

Fig. 1. Localização do Reservatório de Salto Segredo, com indicações das estações de amostragem.

\section{Métodos}

As capturas dos exemplares foram realizadas mensalmente no período de março/93 a fevereiro/94. O esforço aplicado foi de 24 horas, com despescas nos períodos noturno-matutino (8:00 às 9:00 h), diurno (16:00 às 18:00 h) e vespertinonoturno (22:00 às 23:00 $\mathrm{h}$ ).

Os equipamentos de captura empregados consistiram de redes de espera simples com $20 \mathrm{~m}$ de comprimento e malhas de 3 a $14 \mathrm{~cm}$ entre nós adjacentes e redes tresmalho com malhas de 6 a $8 \mathrm{~cm}$ entre nós adjacentes.

Após obtenção dos dados biométricos dos 1590 exemplares capturados, os 211 estômagos com conteúdo foram fixados em formol $4 \%$ para as análises qualitativas e quantitativas do material ingerido. $\mathrm{O}$ tamanho dos exemplares que continham alimento no estômago variou de 8,2 à $26,0 \mathrm{~cm}$ de comprimento padrão. 
Nestas análises foram empregados os métodos de freqüência de ocorrência, numérico e gravimétrico (HYNES 1950; HYSLOP 1980). Os dados resultantes foram combinados no indice de importância relativa (IRI) (PINKAS 1971). Estes foram transformados em porcentagens, as quais foram ordenadas em forma decrescente e efetuadas somatórias cumulativas, após o que os itens foram classificados em: preferenciais - próximo de 50\%; secundários - entre $50 \%$ e $75 \%$ e acessórios os demais itens (ROSECCHI \& NOUAZE 1987).

Com o objetivo de verificar se ocorreram ou não mudanças na dieta de $O$. longirostris com relação às épocas do ano e ambientes de coletas, foi aplicado o índice de similaridade de Bray-Curtis (KREBBS 1989).

As variações ontogenéticas na dieta foram avaliadas através das freqüências relativas de cada item para cada classe de comprimento padrão de $O$. longirostris. Os comprimentos máximo, médio e mínimo dos peixes-presa para as diferentes classes de comprimento padrão do predador, foram obtidos considerando-se todas as presas não digeridas encontradas nos estômagos.

Para o estabelecimento da relação entre o comprimento padrão da presa e o comprimento padrão do predador, utilizou-se a seguinte equação:

$$
C R P=\left(L s_{\text {presa }} / L s_{\text {predador }}\right)
$$

onde: (CRP) comprimento relativo da presa, (Ls presa) comprimento padrão da presa, (Ls predador) comprimento padrão do predador

Além de Astyanax sp. 2 e Astyanax sp. 3 encontradas no conteúdo estomacal de $O$. longirostris, outras cinco espécies do gênero Astyanax foram capturadas no reservatório de Salto Segredo durante o período de coletas. Todas estão sendo revisadas pelo Dr. Júlio César Garavello (Universidade Federal de São Carlos), por este motivo, optou-se por codifica-las com algarismos arábicos.

\section{RESULTADOS}

Através do indice de importância relativa, aplicado para o total de estômagos analisados, evidenciou-se que o alimento principal de $O$. longirostris foi Astyanax sp. 2, sendo os demais considerados acessórios (Fig. 2A).

Esta mesma análise, feita para os diferentes locais de coleta, revelou que no reservatório Astyanax sp. 2 destacou-se como presa preferencial representando $87,74 \%$ da dieta, enquanto todos os outros itens foram considerados acessórios. Nos tributários, Astyanax sp. 2 continuou sendo o item principal, no entanto, sua participação na dieta caiu para $53,16 \%$. Assim, Astyanax sp. 3 com 19,57\%, Hemiptera com $9,20 \%$ e Pimelodus sp. com 7,80\% foram consideradas presas secundárias e as demais acessórias. A jusante, apenas quatro estômagos foram analisados, sendo que Astyanax sp. 3 representou $71,96 \%$ da dieta e Hymenoptera 28,04\%, (Fig. 2A).

Em relação às diferentes estações do ano nota-se que no outono Astyanax sp. 2 foi considerado item principal, no entanto, sua representatividade foi de $58,66 \%$, sendo sua dieta complementada por Hemiptera (18,19\%) e Pimelodus sp. $(15,34 \%)$. Nesta estação, os outros nove itens foram considerados acessórios. No 
inverno, observou-se redução na variedade de itens alimentares (seis), Astyanax sp. 2 continuou como item principal e representou $81,28 \%$ destes e Astyanax sp. 3 $18,17 \%$. Na primavera, apesar do maior número de itens alimentares (13), Astyanax sp. 2 foi alimento praticamente exclusivo $(99,70 \%)$, sendo os demais itens acessórios. Dos 10 itens registrados no verão, Astyanax sp. 2 (73,18\%), foi também item principal, entretanto, Astyanax sp. 3 contribuiu com 13,19\% da dieta (Fig. 2B).

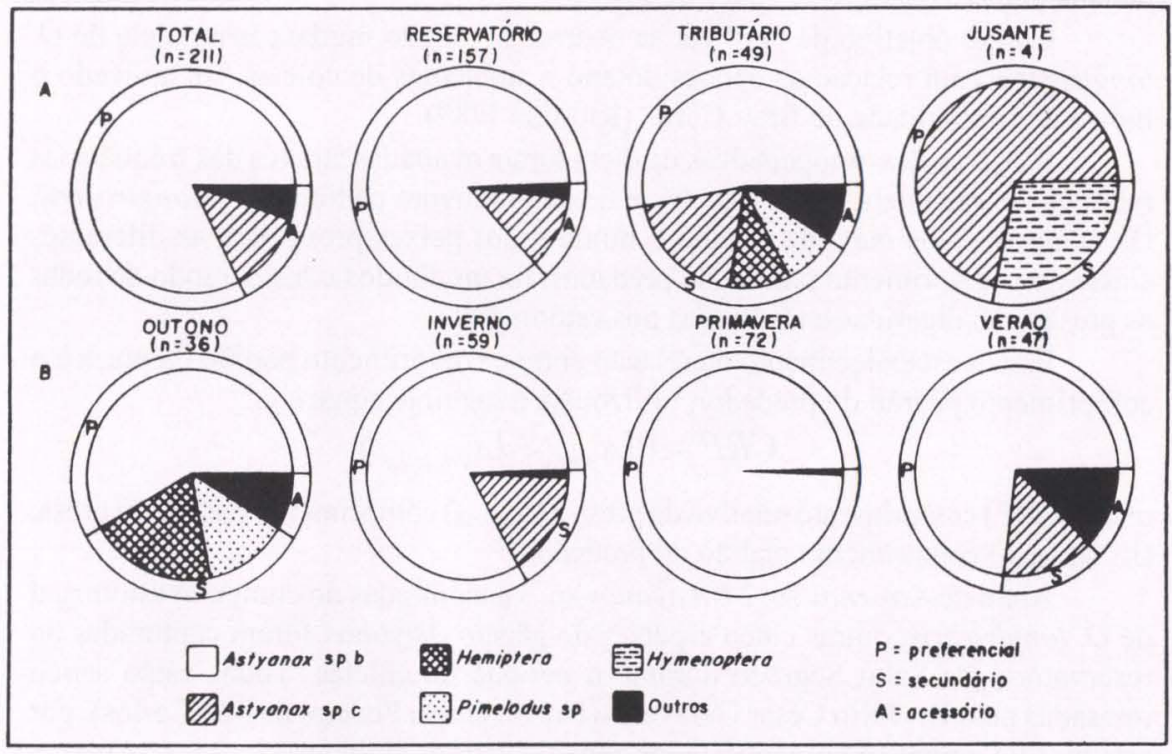

Fig. 2. Participação relativa dos itens alimentares na dieta de Oligosarcus longirostris, no reservatório de Salto Segredo: (A) para o total capturado e diferentes ambientes de coleta; (B) para as diferentes estações do ano.

Os padrões de similaridade da dieta de $O$. longirostris nos diferentes locais de coletas e estações do ano estão representados na figura 3. Considerando-se o nível da distância de Bray-Curtis de aproximadamente 0,16 , dois grandes grupos são formados, o que diferencia de um modo geral o reservatório dos tributários e jusante.

O coeficiente de correlação cofenético foi igual a 0,93 indicando que o dendrograma obtido é uma boa representação da matriz de similaridade original.

Variações consideráveis na dieta foram observadas quando analisados exemplares de diferentes tamanhos (Fig. 4). Nota-se que indivíduos de 8.2 à $9,0 \mathrm{~cm}$ consumiram apenas insetos. Deste, até o tamanho de $17,0 \mathrm{~cm}$, além de insetos, os peixes fizeram parte da dieta. Crustáceos (Aegla sp.) estiveram presentes na alimentação dos indivíduos de 11,0 a $15,0 \mathrm{~cm}$. A partir de $17,0 \mathrm{~cm}$ O. longirostris alimentou-se exclusivamente de peixes.

As relações entre os comprimentos da presa e do predador são apresentados na figura 5A. Verificou-se que conforme os exemplares crescem, ocorre concomitantemente, leve acréscimo no tamanho das presas. No entanto, apesar de ocorrer 
incremento de presas maiores na dieta dos predadores maiores, presas menores também foram ingeridas (Fig. 5A). Analisando-se o comprimento relativo das presas (Fig. 5B), observa-se que os indivíduos menores, até o tamanho aproximado de $14,0 \mathrm{~cm}$, ingeriram, proporcionalmente ao seu tamanho, presas maiores.

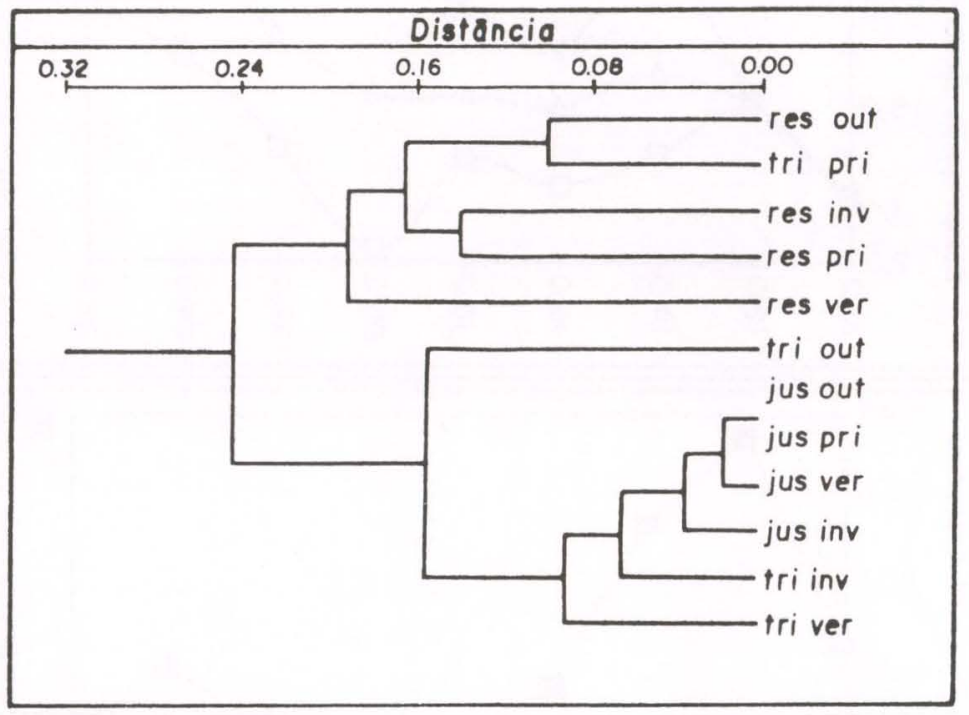

Fig. 3. Dendrograma representativo das relações de similaridade entre as dietas de Oligosarcus longirostris nos diferentes ambientes de coleta.

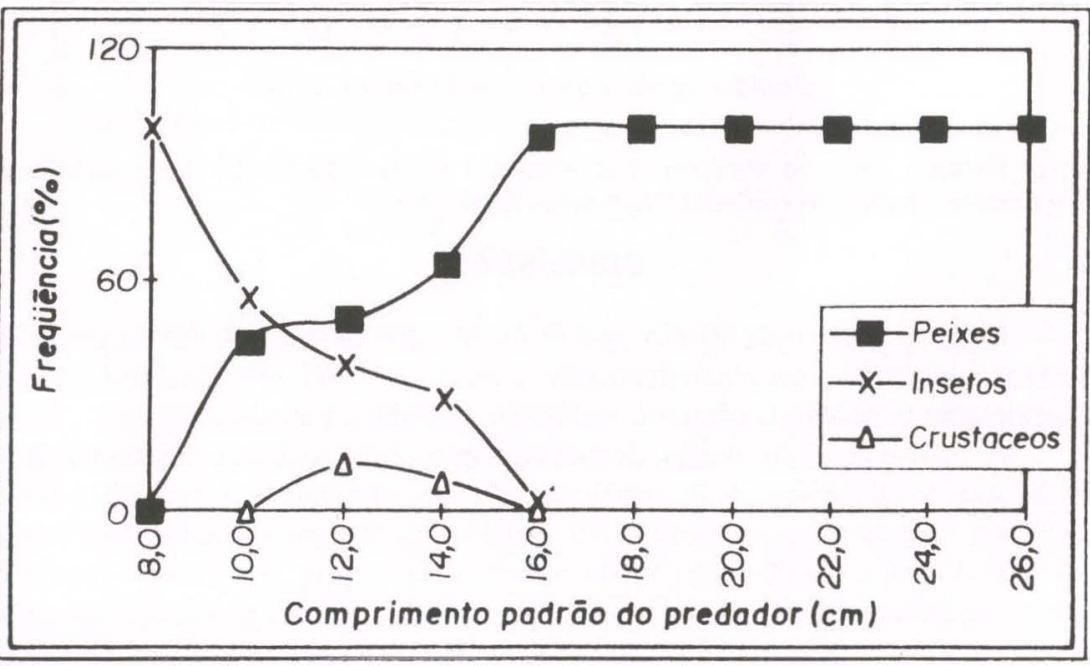

Fig. 4. Freqüência dos itens alimentares ingeridos por Oligosarcus longirostris conforme seu comprimento padrão. 

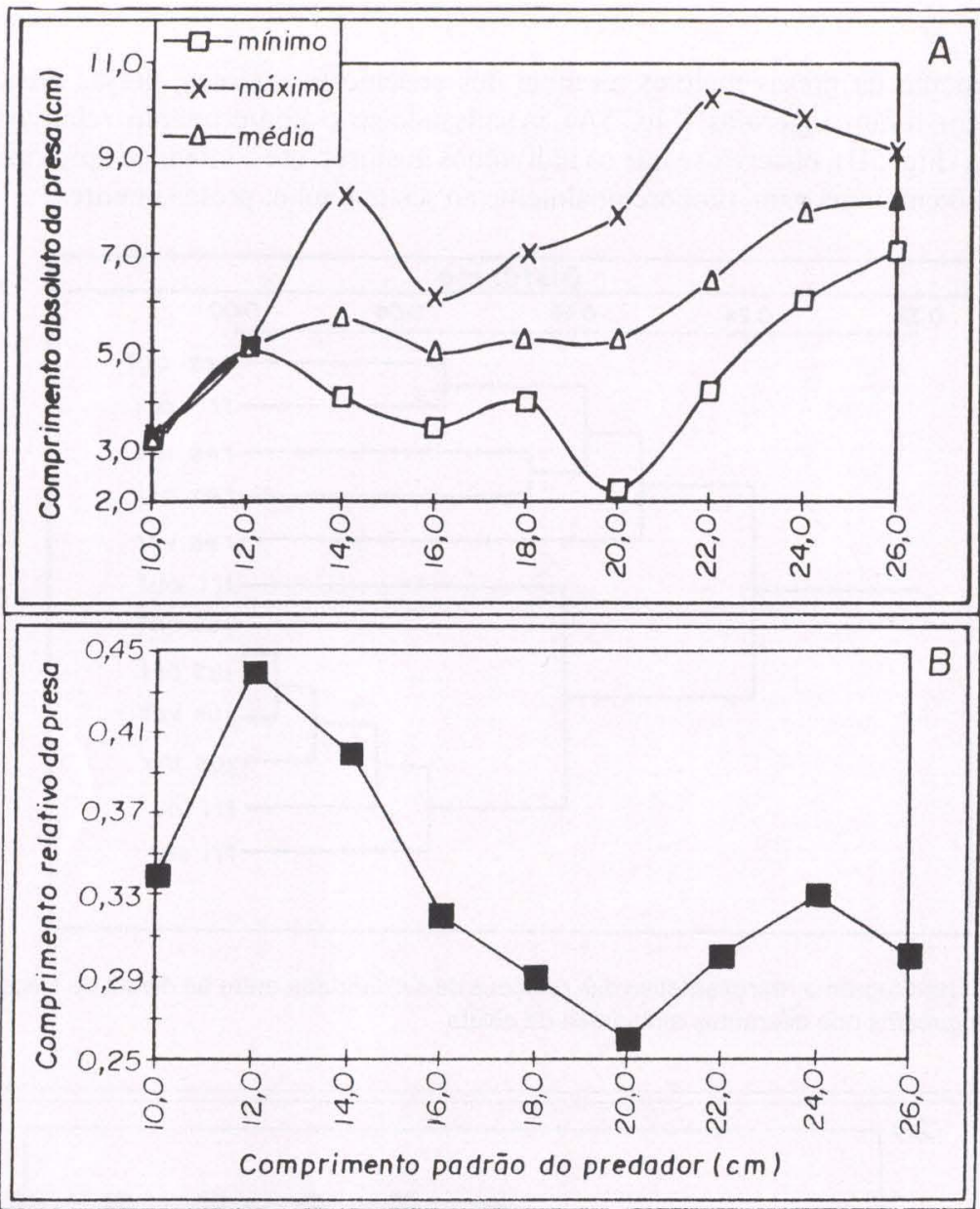

Fig. 5. Relação entre os comprimentos absolutos (A) e relativos (B) das presas e os comprimentos padrão do predador Oligosarcus longirostris.

\section{DISCUSSÃO}

Constatou-se, neste estudo, que $O$. longirostris apresentou hábito alimentar carnívoro passando a ser exclusivamente ictiófago aos $16,0 \mathrm{~cm}$, tamanho este que coincide com tamanho da primeira maturação gonadal para esta espécie.

A análise geral dos dados, demonstrou que a principal fonte alimentar de $O$. longirostris foi Astyanax sp. 2, principalmente nos ambientes reservatório e tributário. Isto, no entanto, parece estar relacionado mais à disponibilidade da presa no ambiente do que ao caráter seletivo da espécie, uma vez que esta presa foi considerada a espécie mais abundante na pesca experimental, realizada paralelamente a este estudo (AGOSTINHO et al. 1997), principalmente no reservatório. Evidências do caráter oportunista desta espécie foram também observados a jusante, onde o item principal passou a ser Astyanax sp. 3, considerado abundante neste ambiente. 
Não foram constatadas diferenças consideráveis na dieta da espécie com relação ao seu item preferencial (Astyanax sp. 2), nas distintas épocas do ano, sendo constatadas apenas alterações nas proporções deste item. Pela análise de Cluster, observou-se muito mais uma diferenciação espacial que temporal na dieta desta espécie. Tributário-primavera uniu-se ao ambiente reservatório, principalmente em função da elevada freqüência de Astyanax sp. 2 ingerido por $O$. longirostris nesta estação do ano quando a espécie presa predominou em todos os ambientes de coletas. Segundo LowE MCCONNELL (1987), as variações na dieta de um predador podem ser decorrentes de significativas flutuações abióticas, sazonais ou a mudanças na abundância da espécie presa, pois a disponibilidade é um fator chave na determinação do que é ingerido pelo peixe, embora possa ou não determinar a dieta (LAGLER et al. 1977). A este respeito, WELCOME (1979) e MOYLE \& CECH (1988) comentam que, apesar da considerável especialização anatômica exibida por algumas espécies, a dieta dos peixes parece estar mais condicionada à disponibilidade de alimento no ambiente.

Nenhum caso de canibalismo foi registrado para esta espécie durante o período de estudos. Segundo NIKOLSKY (1963), em muitos casos este tipo de comportamento atua para regular a abundância e reduzir o agravamento das relações alimentares que podem ocorrer como resultado da superpopulação, fato que não deveria estar ocorrendo no início da formação do reservatório.

Distintas mudanças foram observadas na alimentação de $O$. longirostris conforme o crescimento. $\mathrm{O}$ item Artropodes, predominante na dieta dos jovens foi sendo gradativamente substituído por peixes, passando a ter uma dieta exclusivamente ictiófaga quando adultos. A este respeito, WootTon (1990) comenta que o primeiro ano de vida de um peixe é de rápido crescimento e que este é preferencialmente o período em que a dieta muda rapidamente. Estas mudanças ontogenéticas somam-se às mudanças morfológicas e maturacionais, particularmente devido ao aumento da boca e da habilidade locomotora. NIKOLSKY (1963) comenta que estes fatores podem estar relacionados ao próprio desenvolvimento morfológico dos peixes, ou ainda, à ocupação de estratos diferentes conforme a fase de desenvolvimento, como por exemplo, os jovens se posicionarem mais nas regiões marginais e os adultos nas áreas mais abertas onde as ofertas de alimento são diferentes.

Com relação ao tamanho das presas ingeridas, verificou-se que indivíduos menores de $O$. longirostris ingeriram indivíduos maiores em relação ao seu tamanho, entretanto os maiores exemplares dificilmente apresentavam apenas uma presa no estômago. Popova (1978) verificou que embora as curvas que representem o tamanho absoluto da presa sejam ascendentes conforme é ampliado o tamanho do predador, o tamanho relativo da presa cai quando da transição para a fase piscívora. PITCHER \& HART (1982) comentam que deve existir um tamanho ótimo de presa no qual ocorre o maior ganho líquido de energia para o predador, visto que o consumo de presas muito pequenas ou muito grandes acarreta um custo semelhante para o peixe. PIANKA (1982) observa, no entanto, que qualquer consumidor ótimo prefere gastar sua energia em encontrar e capturar alimentos que rendam muito energicamente por unidade de consumo. O indivíduo com uma estratégia ótima para conseguir alimento deve aproveitar as rotas alimentares naturais sem perder tempo

Revta bras. Zool. 15 (4): 985 - 993, 1998 
e energia buscando presas situadas em lugares e em tempos inadequados. Este autor salienta ainda que, o que é ótimo em um ambiente, raras vezes o é em outro, e que a anatomia de um animal limita muito a tática ótima de obtenção de alimento. HAHN et al. (no prelo), ALMEIDA et al. (no prelo) e LOUREIRO \& HAHN (1996) constataram que as espécies de piscívoros por eles estudadas não demostraram uma seleção muito rígida com relação ao tamanho das presas, fato constatado também para esta espécie o que provavelmente esteja relacionado à disponibilidade de presas no local e/ou a facilidade de captura suprindo a falta de presas maiores pelo maior número de presas.

AgRAdECIMENTOS. Agradecemos ao Nupelia (Núcleo de Pesquisas em Limnologia, Ictiologia e Aqüicultura) pelo apoio logístico, ao Convênio UEM/COPEL pelo apoio financeiro, ao Prof. Dr. Angelo A. Agostinho pela oportunidade e a Vera. Lúcia L. Almeida pela leitura e sugestões.

\section{REFERÊNCIAS BIBLIOGRÁFICAS}

Agostinho, A.A.; L.M. Bini; L.C. Gomes. 1997. Ecologia de comunidades de peixes da área de influência do reservatório de Segredo, p.97-111. In: A.A. Agostinho \& L.C. GOMES (Ed.): Reservatório de segredo-bases para o manejo. Maringá, Eduem, 386p.

Almeida, V.L.L. DE; N.S. Hahn; A.E.A.M. VAZzoler. (no prelo). Feeding patterns in five predatory fishes of the high Paraná River floodplain (PR, Brazil). Ecology of Freshwater Fish.

HAHN, N.S.; A.A. AgostinHo; R. GoITEIN. (no prelo). Feeding ecology of curvina Plagioscion squamosissimus (Heckel, 1840) (Pisces, Perciformes) in the Itaipu Reservoir and Porto Rico floodplain. Acta Limnologica Brasiliensia.

HYNES, H.B.N. 1950. The food of freshwater sticklebacks (Gasterosteus aculeatus and Pigosteus pungitius), with a review of methods used in studies of the food of fishes. Jour. Anim. Ecol. 19 (1): 36-35.

HYSLOP, E.J. 1980. Stomach contents analysis, a review of methods and their application. Jour. Fish Biol. 17: 411-429.

KREBBS, C.J. 1989. Ecologycal methodology. New York, Harper Collins Publishers, 654p.

LAGler, K.F.; J.E. BARdACH; R.R. Miller; D.R.M. PASSINO. 1977. Ichtyology. New York, John Wiley \& Sons, $2^{\text {nd }}$ ed., 506p.

LOUREIRO, V.E.; N.S. HAHN. 1996. Dieta e atividade alimentar da traíra, Hoplias malabaricus (Bloch, 1794) (Osteichthyes, Erythrynidae), nos primeiros anos de formação do reservatório de Segredo-PR. Acta Limnologica Brasiliensia 8: 195-205.

LOWE-MCCONELL, R.H. 1987. Ecological studies in tropical fish communities. Cambridge, University Academic Press., 382p.

MOYLE, P.B. \& J.J. CECH JR. 1988. Fishes: an introduction to ichthyology. Englewood Cliffs, Prentice Hall, $2^{\text {nd }}, 559 \mathrm{p}$.

NIKOLSKY, G.V. 1963. The ecology of fishes. London, Academic Press, 352p.

PIANKA, E.R. 1982. Ecologia evolutiva. Barcelona, Omega, 312p.

PINKAS, L. 1971. Food habits study, p. 5-10. In: L. PINKAS; M.S. OLIPHANT; I.L.K. 
IVERSON (Ed.). Food habits of albacore bluefin tuna, and bonito in California waters. Fish Bull. 152: 1-105.

Pitcher, T.J. \& P.J.B. HART. 1982. Fisheries ecology. London, Croom Helm, $414 \mathrm{p}$.

Popova, A.O. The role of predaceous fish in ecosystems, p.215-249. In: S.D. GERKING (Ed.). Ecology of Freshwater Fish Production. Oxford, Blackwell Scientific, 520p.

Rosecchi, L. \& Y. NouAze. 1987. Comparaison de cinq indices alimentaires utilisés dans l'analyse des contenus stomacaux. Rev. Trav. Pèches mar. 49 (4): 111-123.

WELCOME, R.L. 1979. Fisheries ecology of floodplain rivers. London, Longman, $317 \mathrm{p}$.

Wooton, R.J. 1990. Ecology of teleost fishes. London, Chapman and Hall, 404p.

Recebido em 19.IX.1997; aceito em 17.XI.1998. 